\title{
DNA methylation patterns associated with oxidative stress in an ageing population
}

Åsa K. Hedman', Mihkel Zilmer², Johan Sundström, Lars Lind ${ }^{3}$ and Erik Ingelsson ${ }^{1,4^{*}}$

\begin{abstract}
Background: Oxidative stress has been related to type 2 diabetes (T2D) and cardiovascular disease (CVD), the leading global cause of death. Contributions of environmental factors such as oxidative stress on complex traits and disease may be partly mediated through changes in epigenetic marks (e.g. DNA methylation). Studies relating differential methylation with intermediate phenotypes and disease endpoints may be useful in identifying additional candidate genes and mechanisms involved in disease.

Methods: To investigate the role of epigenetic variation in oxidative stress marker levels and subsequent development of CVD and T2D, we performed analyses of genome-wide DNA methylation in blood, ten markers of oxidative stress (total glutathione [TGSH], reduced glutathione [GSH], oxidised glutathione [GSSG], GSSG to GSH ratio, homocysteine $[\mathrm{HCY}]$, oxidised low-density lipoprotein (oxLDL), antibodies against oxLDL $[O L A B]$, conjugated dienes [CD], baseline conjugated dienes [BCD]-LDL and total antioxidant capacity [TAOC]) and incident disease in up to 966 age-matched individuals.
\end{abstract}

Results: In total, we found 66 cytosine-guanine $(\mathrm{CpG})$ sites associated with one or more oxidative stress markers (false discovery rate [FDR] <0.05). These sites were enriched in regulatory regions of the genome. Genes annotated to CpG sites showed enrichment in annotation clusters relating to phospho-metabolism and proteins with pleckstrin domains. We investigated the contribution of oxidative stress-associated CpGs to development of cardiometabolic disease. Methylation variation at CpGs in the 3'-UTR of HIST1H4D (cg08170869; histone cluster 1, H4d) and in the body of DVL1 (cg03465880; dishevelled-1) were associated with incident T2D events during 10 years of follow-up (all permutation p-values $<0.01$ ), indicating a role of epigenetic regulation in oxidative stress processes leading to development or progression of diabetes. Methylation QTL (meQTL) analysis showed significant associations with genetic sequence variants in cis at 28 (42\%) of oxidative stress phenotype-associated sites (FDR $<0.05$ ). Integrating cis-meQTLs with genotypephenotype associations indicated that genetic effects on oxidative stress phenotype at one locus (cg07547695; BCL2L11) may be mediated through DNA methylation.

Conclusions: In conclusion, we report novel associations of DNA methylation with oxidative stress, some of which also show evidence of a relation with T2D incidence.

Keywords: DNA methylation, Epigenetics, Oxidative stress, Type 2 diabetes, Cardiovascular disease

\footnotetext{
* Correspondence: eriking@stanford.edu

'Department of Medical Sciences, Molecular Epidemiology and Science for

Life Laboratory, Uppsala University, Uppsala, Sweden

${ }^{4}$ Department of Medicine, Division of Cardiovascular Medicine, Stanford

University School of Medicine, Stanford, CA, USA

Full list of author information is available at the end of the article
}

(c) The Author(s). 2016 Open Access This article is distributed under the terms of the Creative Commons Attribution 4.0 International License (http://creativecommons.org/licenses/by/4.0/), which permits unrestricted use, distribution, and reproduction in any medium, provided you give appropriate credit to the original author(s) and the source, provide a link to the Creative Commons license, and indicate if changes were made. The Creative Commons Public Domain Dedication waiver (http://creativecommons.org/publicdomain/zero/1.0/) applies to the data made available in this article, unless otherwise stated. 


\section{Background}

CVD is the most common cause of death globally. Risk factors of CVD include high blood pressure, smoking, hyperglycaemia, T2D and obesity [1]. Previous studies have suggested that increased oxidative stress as a consequence of obesity and T2D may contribute to the increased risk of CVD [2,3]. Furthermore, CVD and risk factors of CVD have been associated with changes in levels of oxidative stress markers $[4,5]$.

Some of the oxidative markers have also been associated with sub-phenotypes of CVD and T2D. For example, oxLDL, elevated in CVD $[6,7]$, plays a role in atherosclerosis through its role in maturation of macrophages contributing to inflammation and foam formation [8, 9]. Furthermore, oxLDL is associated with insulin resistance [10] indicating a role of oxidative stress in progression to insulin resistance and T2D. The amino acid HCY has been associated with inflammation in blood vessels, progression to atherosclerosis and development of CVD, particularly ischemic stroke [11, 12]; however, the causal role of homocysteine has been challenged [13-15].

While genome-wide association studies (GWAS) have been successful in identifying numerous common genetic sequence variants associated with metabolic disease and CVD [16-20], so far these only explain a small proportion of the variability of these phenotypes. In addition, environmental factors also influence disease susceptibility. Such contributions may be partly mediated through changes in epigenetic marks (e.g. DNA methylation), affecting transcription through mechanisms independent of DNA sequence [21]. Thus, studies relating differential methylation with intermediate phenotypes and disease endpoints may be useful in identifying additional candidate genes and mechanisms involved in these diseases [22]. Previous studies support a role for DNA methylation in common complex diseases [23-26] and in mediation of environmental exposures of importance for CVD and T2D, such as cigarette smoking $[27,28]$ and oxidative stress $[29,30]$.

In this study, we aimed to examine epigenetic variation in blood cells in relation to oxidative stress and development of T2D and CVD. Blood-derived cells play a role in several processes relating to cardiometabolic disease [31, 32]. Furthermore, previous studies have shown methylation variation in blood to reflect differential methylation in various tissues [33-37]. We performed analyses of genome-wide DNA methylation, ten markers of oxidative stress (TGSH, GSH, GSSG, GSSG/GSH ratio, HCY, oxLDL, OLAB, CD, BCD-LDL and TAOC) and incident disease in 966 individuals from the general population.

\section{Methods}

\section{Study sample}

The Prospective Investigation of the Vasculature in Uppsala Seniors (PIVUS) is a prospective community- based cohort of participants from Uppsala, Sweden. All men and women at age 70 living in Uppsala in 2001 were invited to participate. The 1016 participants $(50 \%$ women) have been extensively phenotyped, as described previously [38], and on the Internet (www.medsci.uu.se/ pivus/). The participants have been re-examined at ages 75 and 80, and their morbidity and mortality has been followed via national registers and journal review. Clinical diagnoses by journal review of CVD and/or T2D at 80 years (10 years after baseline) were used to define disease events. For analysis of CVD outcomes, we included myocardial infarction (ICD-10 code: I21), stroke (ICD-10 code:I63) and heart failure (ICD-10 code: I50). During the 10 year follow up period (between ages 70 and 80), there were 142 deaths; 34 of these due to CVD related disease.

\section{Markers of oxidative stress}

The methods of collection and validation of oxidative markers have previously been described [5]. Briefly, TGSH, GSH, CD and TAOC were determined using a method described and validated in Annuk et al. [39]. HCY levels were measured using an Enzyme Immunoassay method (Axis-Shield Diagnostics Ltd, UK). BCD-LDL were measured using a method described in detail in [40]. Enzyme-linked immuno-absorbent assays were used to determine levels of serum oxLDL (Mercodia AB, Sweden) and OLAB (BioMedica, Austria).

\section{Genome-wide DNA methylation profiling}

Blood for the DNA methylation assay was collected at the baseline examination. Genomic DNA was extracted from blood samples and bisulphite conversion of $500 \mathrm{ng}$ genomic DNA was performed using the EZ-96 DNA Methylation Gold Kit (Zymo Research Product, Germany). The equivalent of approximately $200 \mathrm{ng}$ of bisulphite converted DNA was removed, evaporated to a volume of $<4 \mu \mathrm{l}$, and used for methylation profiling using the Illumina Infinium assay and the Illumina HumanMethylation450_v.1.2 bead chip according to the protocol from the supplier (Illumina Inc., San Diego, CA, USA). The results were analysed with GenomeStudio 2011.1 (Illumina Inc., San Diego, CA, USA). After exclusion of replicates, a total of 1002 study participants had methylation data available for quality control procedures. Three samples were excluded based on poor bisulphite conversion efficiency, twelve samples due to low pass rate of $\mathrm{CpG}$ sites $(<98.5 \%$ with a detection $P$ value $>0.01$ ) and a further six samples based on low SNP genotype match ( $>1$ SNP mismatches) between genotypes from the methylation array and Omni/Metabochip genotyping chips leaving 981 samples. Following additional removal of participants with high leukocyte cell counts $\left(>10 \times 10^{9}\right.$ cells $\left./ L ; n=14\right)$ and one individual with no data on oxidative stress markers, 966 individuals 
remained for downstream analysis. The signal intensities for the methylated and unmethylated states were then quantile normalised for each probe type separately, and beta values were calculated. Mapping and annotation of the 485,764 probes on the HumanMethylation450K BeadChip has previously been described [41]. Briefly, probes mapping to multiple locations (with at least two mismatches) in the human reference genome (GRCh37) were excluded leaving 459,433 uniquely mapping autosomal probes. Furthermore, probes were filtered based on sequence polymorphisms as follows: those with a common SNP (minor allele frequency $[\mathrm{MAF}]>5 \%$ ) within $10 \mathrm{bp}$ of the methylation site and those overlapping copy number variants were excluded from analysis. This resulted in a final set of 455,127 probes which were then assigned to CpG islands and RefSeq transcripts downloaded from the UCSC Genome Browser (September, 2012). Probes within $2 \mathrm{~kb}$ away from borders of a CpG island were defined as shores and those within $2 \mathrm{~kb}$ of shores as falling within shelves. The rest were assigned to others/open sea. Probes were mapped in relation to transcripts as follows: TSS1500 (1500 bp to $200 \mathrm{bp}$ upstream of transcriptional start site [TSS]), TSS200 (200 bp upstream of TSS), the 5'-UTR, the first exon, the gene body or the 3'-UTR [42].

\section{Genotyping and imputation}

Individuals were genotyped using the Illumina OmniExpress and Illumina Metabochip microarrays. Prior to imputation, quality control was performed as described below. Exclusion of samples were performed based on the following criteria: genotype call rate $<95 \%$; heterozygosity $>3$ standard deviations (SD); gender discordance; duplicated samples; identity-by-descent match; and ethnic outliers. Monomorphic SNPs; or SNPs with Hardy-Weinberg equilibrium $p$-value $<1$ E10-6; genotype call rate $<0.99$ (SNPs with MAF $<5 \%$ ) or $<0.95$ (SNPs with $\mathrm{MAF} \geq 5 \%$ ); $\mathrm{MAF}<1 \%$ were excluded from analysis. Data were imputed to the 1000G (version: March 2012) multi population reference panel using Impute v.2.2.2 [43]. A plot of the PIVUS data with the data from the multi - population reference panel are included in Additional file 1: Figure S1.

\section{Statistical analyses \\ Association of methylation of blood cell-derived DNA with phenotypes and disease outcomes}

Transformed or raw phenotypes were used (details in Table 1). All models were adjusted for age, sex, batching (clinical visit date), bisulphite conversion efficiency mean (calculated from control probes), bisulphite conversion plate and predicted white cell counts (estimated from the DNA methylation data using the Houseman algorithm [44], as implemented in $\mathrm{R}$ package minfi for Illumina HumanMethylation450 [45], with reference data on sorted blood cell populations from Reinius et al. [46]). To determine whether BMI confounds the relationship between the oxidative marker and DNA methylation, we performed secondary models additionally adjusted for BMI for those oxidative markers that showed association with BMI in sex adjusted models (nominal $p$-value $<0.01$ ). For continuous phenotypes, the associations between normalised DNA methylation beta values and phenotypes were modelled by a linear model, using R [47] and the $l m$ function, fitted by maximum-likelihood assuming a normally distributed error term. For binary phenotypes (case/control), we fitted a logistic regression in $\mathrm{R}$ using the $\mathrm{glm}$ function (binomial family [link function, logit]), to model the association between standardised DNA methylation and case/control status. Disease was used as the outcome variable, and technical covariates (as above), age, sex, predicted white cell count and standardised methylation as independent variables as follows: Disease status $(1 / 0) \sim$ standardised methylation + age + sex + predicted white cell counts + technical covariates. In secondary models, we also included BMI and smoking as covariates in the model. In all cases, a likelihood ratio test was used to assess the significance of the phenotype effect. The $p$-value of the phenotype effect in each model was calculated from the Chi-square distribution with 1 degree of freedom using $-2 \log$ (likelihood ratio) as the test statistic. FDR were estimated based on Q-values [48]. For CVD and T2D outcomes, significance was assessed using permutations of case/control status (10,000 permutations). A permutation $p$-value of $<0.01$ was considered as significant.

\section{meQTL and SNP-phenotype analyses}

Associations between normalised DNA methylation beta values and genotypes were modelled by a linear model, using $\mathrm{R}$ [47] and the $l m$ function, fitted by maximumlikelihood assuming a normally distributed error term. We assumed an additive genetic model. A likelihood ratio test was used to assess the significance of the SNP effect. The $p$-value of the SNP effect in each model was calculated from the Chi-square distribution with 1 degree of freedom using -2log(likelihood ratio) as the test statistic. We only performed cis analysis, which was limited to SNPs located within $100 \mathrm{~kb}$ either side of the probe location. To perform analyses in $\mathrm{R}$, genotype probabilities (from IMPUTE) were transformed to posterior mean genotypes (MACH format [49]). Further, we only included SNPs with a MAF $<5 \%$ and INFO (from imputation) $>0.8$ in down-stream analyses. Since we did not perform a array-wide cis-meQTL scan, and were therefore concerned of potential bias in the $p$-value distribution, we estimated FDR from permutations, rather than using Q-values. We permuted SNP data, performed cis-meQTL analysis on the permuted data, and repeated 
Table 1 Methylation sites associated with oxidative markers in blood

\begin{tabular}{|c|c|c|c|c|c|}
\hline Phenotype & Number & Mean (SD) & Range & $\begin{array}{l}\text { No. of } \mathrm{CpG} \text { sites associated } \\
\text { at FDR }<0.05\end{array}$ & $\begin{array}{l}\text { No. of } \mathrm{CpG} \text { sites associated } \\
\text { at } P \text {-value }<1.1 \mathrm{E}-7\end{array}$ \\
\hline TGSH $(\mu \mathrm{g} / \mathrm{ml})$ & 958 & 905.0 (199.3) & $440-1836$ & 18 & 3 \\
\hline $\mathrm{GSH}(\mu \mathrm{g} / \mathrm{ml})$ & 957 & 829.9 (191.1) & $398-1730$ & 25 & 2 \\
\hline GSSG $(\mu \mathrm{g} / \mathrm{ml})$ & 959 & $75.8(35.7)$ & $23-257$ & 1 & 1 \\
\hline GSSG/GSH ratio & 957 & $0.1(0.06)$ & $0.03-0.53$ & 21 & 3 \\
\hline $\mathrm{HCY}(\mu \mathrm{mol} / \mathrm{l})$ & 966 & $10.6(3.98)$ & $3.4-40.7$ & 9 & 6 \\
\hline In OxLDL (U/I) & 966 & $132.8(47.7)$ & $42-285$ & 1 & 1 \\
\hline $\mathrm{CD}(\mu \mathrm{mol} / \mathrm{l})$ & 966 & 40.7 (11.1) & $13.8-90.4$ & 1 & 1 \\
\hline BCD-LDL $(\mu \mathrm{mol} / \mathrm{l})$ & 964 & $21.7(7.4)$ & $7.8-71.7$ & 6 & 4 \\
\hline In OLAB (U/I) & 734 & $5.9(0.96)$ & $3.4-7.9$ & 0 & 0 \\
\hline TAOC (\%) & 957 & $37.6(3.9)$ & $22-55$ & 0 & 0 \\
\hline
\end{tabular}

$S D$ standard deviation, $C P G$ cytosine-guanine site, FDR false discovery rate, $T G S H$ total glutathione level, GSH reduced glutathione, GSSG oxidised glutathione, $H C Y$ homocysteine, OxLDL oxidised LDL, CD conjugated dienes, $B C D$ baseline CD, OLAB antibodies against oxLDL, TAOC total antioxidant capacity

this for ten replicates selecting the most associated SNP per methylation probe in each round of permutation. FDR of 0.05 was calculated as the nominal $p$-value threshold which gave less than $5 \%$ significant associations (i.e. false discoveries) in the permuted data. Associations between oxidative markers and meQTL genotypes were modelled by a linear model, using R [47] and the lm function, fitted by maximum-likelihood assuming a normally distributed error term. We assumed an additive genetic model. A likelihood ratio test was used to assess the significance of the SNP effect. The $p$-value of the SNP effect in each model was calculated from the Chi-square distribution with 1 degree of freedom using -2log(likelihood ratio) as the test statistic. Significance was assessed using permutations of genotype data $(10,000$ permutations). Using a one-sided Fisher exact test we tested for over representation of significant meQTL SNPs in nominally significant GWAS associations $(p$-value $<0.05)$ using GWAS data from the CARDIoGRAM consortium for CHD [20] and the DIAGRAM consortium for T2D [18].

\section{Enrichment in genomic location, regulatory regions, transcription factor binding and biological processes}

Using annotation data described above we tested whether CpGs associated with oxidative markers were enriched in genomic locations with respect to genes and $\mathrm{CpG}$ islands. Enrichment was assessed using a two-sided Fisher exact test. Overlap of associated CpGs with functional regulatory elements across cell types were assessed using data available at RegulomeDB [50]. We determined if the overlap was more than expected by chance by comparing this to random set of CpGs. To assess whether genes annotated to phenotype-associated CpGs are likely to be regulated by a common set of transcription factors (TFs), we utilised PSCAN [51] with the JASPAR database [52]. To place our data in the context of biological processes or pathways, we subjected genes annotated to CpGs associated with phenotypes or genotypes to pathway analysis using DAVID [53, 54] and PANTHER [55-57]. We used only genes on the array as background and considered terms with a $p$-value $<0.05$ following adjustments for multiple testing (to the number of pathways or ontology terms) as significant.

\section{Results}

\section{Identification of DNA methylation patterns of oxidative} stress

We characterised genome-wide blood DNA methylation patterns at 459,235 CpGs mapping uniquely across the genome in 96670 -year old individuals from the PIVUS cohort [38]. We performed genome-wide association scans to determine DNA methylation patterns associated with ten markers of oxidative stress: TGSH, GSH, GSSG, GSSG/GSH ratio, HCY, oxLDL, OLAB, $\mathrm{CD}, \mathrm{BCD}-\mathrm{LDL}$ and TAOC (Table 1). In total, we observed 66 CpGs for which levels of methylation were associated with one or more oxidative markers at aper-trait FDR of $<0.05 ; 18$ of these were also associated with at least one oxidative marker at a Bonferronicorrected alpha threshold $<0.05$ (taking the number of CpGs into account; Additional file 2: Figure S2). Figure 1 shows the associations of $\mathrm{CpGs}$ with oxidative stress markers.

\section{Glutathione}

Glutathione is an important antioxidant and the balance between the reduced and oxidised form is indicative of the oxidative state of an individual. We investigated the association between genome-wide methylation patterns and TGSH, GSH and GSSG and found 18, 25 and one significant CpGs, respectively (FDR $<0.05$; Additional file 3 : Table S1-S3). As should be expected, a large proportion of CpGs associated with TGSH and GSH was shared (14 CpGs; Fig. 1), including sites annotated to BCL2L11 


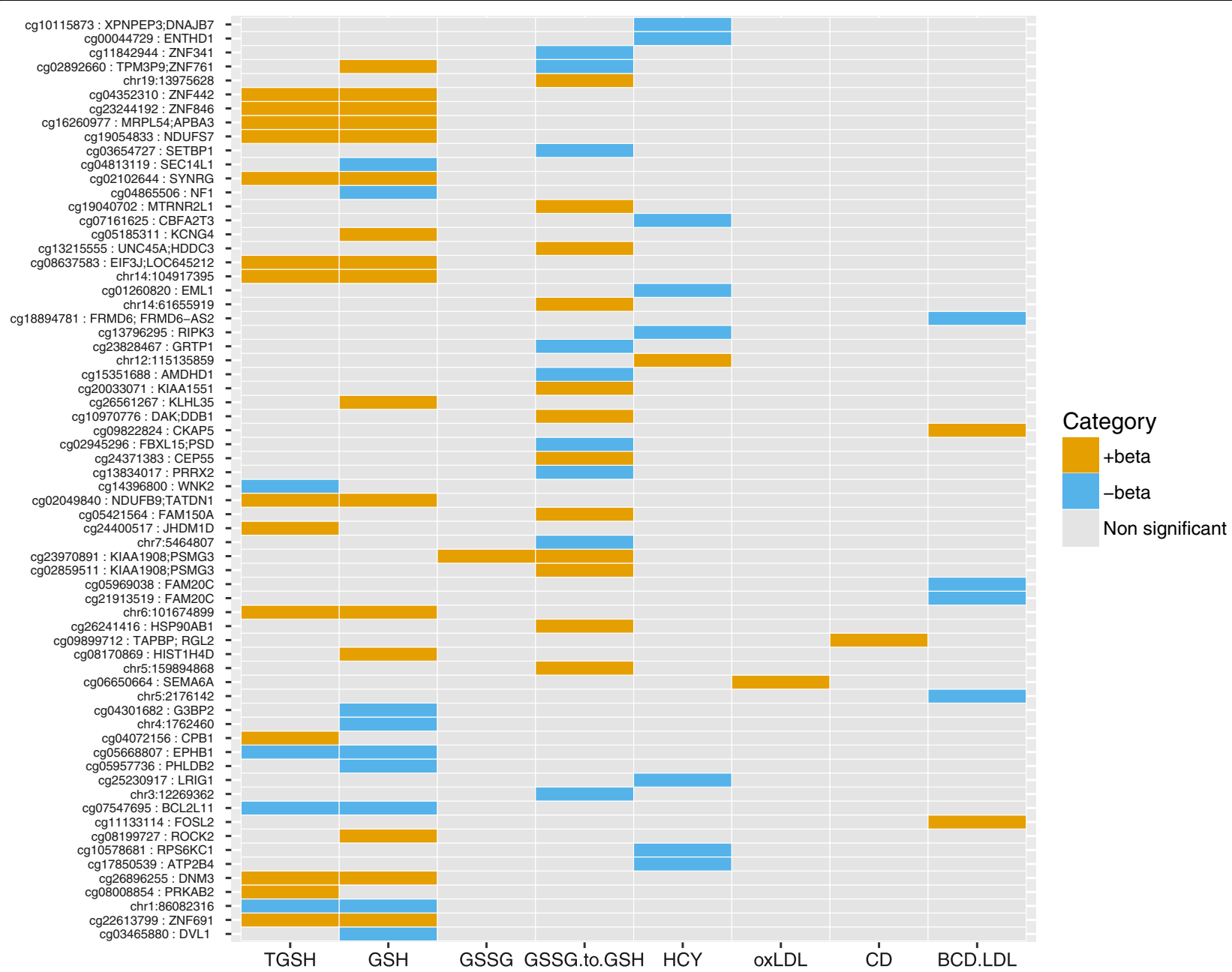

Fig. 1 Overview of CpGs associated with oxidative markers (FDR < 0.05). CpGs are ordered by chromosomal position from bottom (chr. 1) to top (chr. 22). Two of the oxidative markers (OLAB and TAOC) showed no significant associations with CpGs and were not included in the figure

(cg07547695; BCL2-like 11 [apoptosis facilitator]), NDUFS7 (cg19054833; NADH dehydrogenase [ubiquinone] Fe-S protein 7, $20 \mathrm{kDa}$ ) and EIF3J (cg08637583; eukaryotic translation initiation factor 3, subunit J). The ratio of GSSG to GSH can be used as a marker of oxidative stress. We found methylation at $21 \mathrm{CpGs}$ to be significantly associated with $\mathrm{GSSG} / \mathrm{GSH}$ ratio (FDR $<0.05$; Additional file 3: Table S4). Methylation in the promoter region of ZNF761 (cg02892660; zinc finger protein 761) and PSMG3 (cg23970891; proteasome assembly chaperone 3), showed the strongest associations with GSSG/GSH ratio.

\section{Homocysteine}

In our analysis, methylation at nine CpGs were significantly associated with levels of the amino acid HCY (FDR $<0.05$; Additional file 3: Table S5), including sites annotated to genes with a role in signal transduction (ENTHD1 [cg00044729], LRIG1[cg25230917], RIPK3 [cg13796295],
RPS6KC1 [cg10578681], ATP2B4 [cg17850539]) and lipidrelated processes $(R P S 6 K C 1[\mathrm{cg} 10578681])$.

\section{Lipid-related oxidative markers}

In genome-wide methylation analysis, oxLDL was associated with methylation in the 5'-UTR of SEMA6A (cg06650664; sema domain, transmembrane domain [TM], and cytoplasmic domain, [semaphorin] 6A), a protein with anti-angiogenic function $[58,59]$ (FDR $<0.05$; Additional file 3: Table S6). Oxidation of LDL can also be monitored by markers CD and BCD-LDL which measure products of lipid peroxidation. In our analysis, $C D$ was found to associate with higher methylation at a CpG site located in the 3'-UTR of TAPBP (cg09899712; TAP binding protein [tapasin]) and the promoter region (TSS1500) of RGL2 (cg09899712; ral guanine nucleotide dissociation stimulator-like 2 (FDR $<0.05$; Additional file 3: Table S7). Methylation levels at six CpGs were associated with BCDLDL $(F D R<0.05$; Additional file 3: Table S8). These were 
annotated to CKAP5 (cg09822824; cytoskeleton associated protein 5), FOSL2 (cg11133114; FOS-like antigen 2), FAM20C (cg21913519, cg05969038; family with sequence similarity 20, member C) and FRMD6 (cg18894781; FERM Domain containing 6). We observed no overlap in significant $\mathrm{CpGs}$ between the three lipid-related oxidative markers. As CD ( $p$-value $=0.00011)$ and BCD-LDL ( $p$ value $=0.0091)$ were found to be associated with $B M I$ in sex-adjusted models in our study, secondary models adjusted for BMI were also performed for these two phenotypes (Additional file 3: Tables S7-S8). None of the phenotype-associated $\mathrm{CpGs}$ showed a large change in the regression coefficient for the oxidative marker after adjustment with BMI, indicating that BMI did not confound the relationship between DNA methylation and these oxidative markers (Additional file 1: Figure S3).

\section{Functional characterisation of methylation sites associated with oxidative stress}

We explored the functional role of CpGs associated with oxidative markers through investigation of their genomic location with respect to genes, $\mathrm{CpG}$ islands and functional regulatory elements. Phenotype-associated CpGs were enriched in $\mathrm{CpG}$ island shores compared to all CpGs on the array (enrichment $p$-value $=0.04$, Fig. 2). A larger proportion of phenotype-associated CpGs (as compared to all CpGs) were located in promoters of genes $(45 \%$ vs. $36 \%$; enrichment $p$-value $=0.06$, Fig. 3$)$; thus having the potential to affect transcription from adjacent genes. We assessed the overlap of associated CpGs with functional regulatory elements across cell types using RegulomeDB [50]. Twenty-three percentages of sites showed strong evidence of being located in a functional regulatory region (RegulomeDB score 1a-2c;
Additional file 3: Tables S1-S8). This was more than expected by chance (permutation $p$-value $=0.01$ ).

To assess whether genes annotated to phenotypeassociated CpGs are likely to be regulated by a common set of TFs, we used PSCAN [51] with the JASPAR database [52]. We found significant enrichment of four TF binding sites (adjusted $p$-value $<0.05$; Additional file 1 : Table S9), including binding sites of E2F1, a gene/protein with a potential role in atherosclerosis and coronary heart disease (CHD) [60].

To further place our findings in biological context, we performed gene set enrichment analysis [55-57] on the 64 genes annotated to $\mathrm{CpGs}$ associated with oxidative markers. Using the Functional Annotation Clustering Tool in DAVID [53], we found evidence of genes clustering into annotations (enrichment score $>1$ ) relating to functional terms phosphorylation and phosphate/phosphorous metabolic processes and pleckstrin domains (Additional file 1: Table S11).

\section{Genetic associations of oxidative stress-associated DNA methylation}

Genetic sequence variants have been shown to contribute to DNA methylation variation, so called meQTLs [61]. We examined the presence of such loci for the 66 CpGs associated with oxidative markers, and found 28 (41.8\%) of phenotype-CpGs with a significant cis-meQTL (FDR $<0.05$, nominal $p$-value $<2.07 \mathrm{E}-4$, Additional file 1 : Table S12). To assess whether genetic associations with markers of oxidative stress may be mediated by epigenetics, we examined whether genotype-phenotype, genotypeCpG and CpG-phenotype associations overlapped (an overview of the analysis are available in Additional file 4: Figure S4). Overall, we investigated whether cis-meQTL SNPs associated with the oxidative markers and found

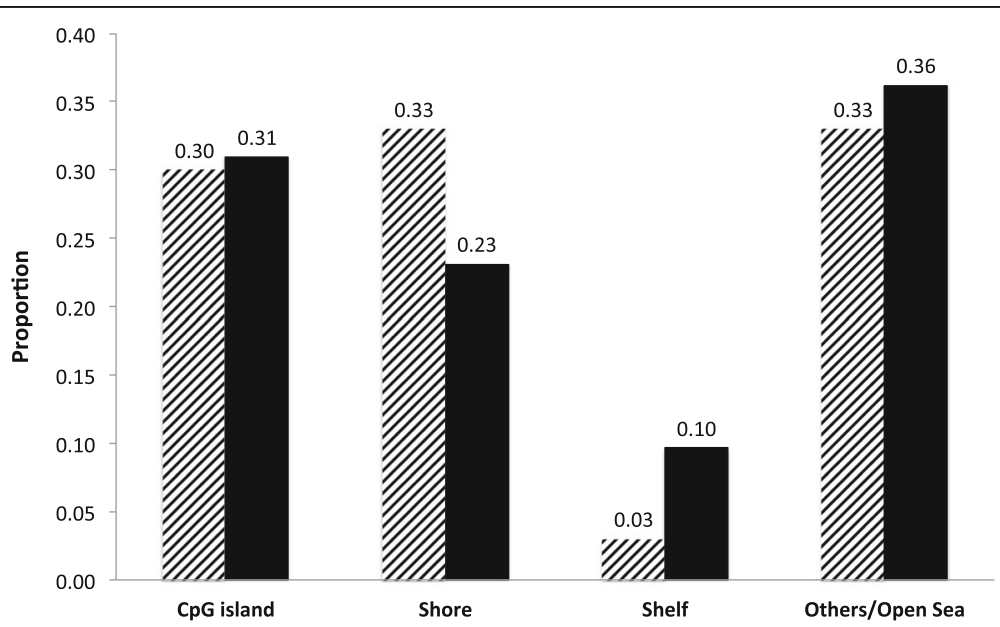

Fig. 2 CpG island context of CpGs associated with oxidative markers. CpGs were classified into: CpG island, Shore, Shelf and Others/Open sea, and phenotype-associated CpGs (striped bars) were compared with all CpGs on the array (black bars). P-values in figure represent results of a one-sided Fisher exact test testing for over- or under-representation of term in either group 


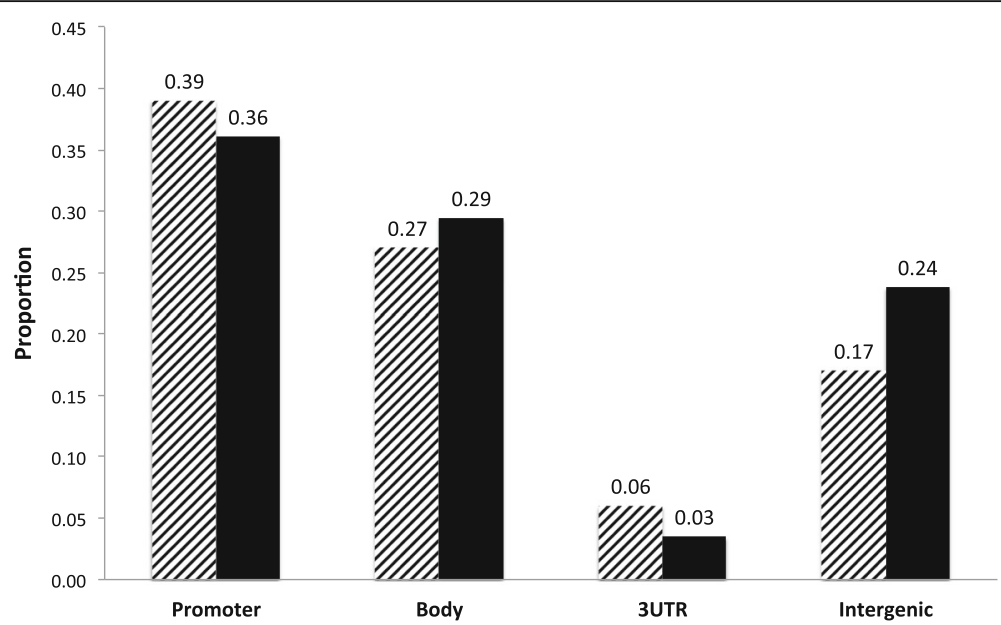

Fig. 3 Genomic distribution of CpGs associated with oxidative markers. CpGs were classified into: promoter (TSS1500, TSS200, 5'-UTR, First exon), Body, 3'-UTR and intergenic. Phenotype-associated CpGs (striped bars) were compared to all CpGs on the array (black bars). P-values in figure represent results of a one-sided Fisher exact test testing for over- or under-representation of term in either group

modest suggestive evidence of association $(p$-value $<0.001)$ for 171 SNPs $(0.56 \%)$ with at least one of the oxidative markers. We found two instances (at $\operatorname{cg} 07547695$ in the 5'-UTR of BCL2L11, associated with both TGSH and GSH) for which associations of genotype-phenotype ( $p$-value <0.001), cis-meQTL $(\mathrm{FDR}<0.05)$ and CpGphenotype $(\mathrm{FDR}<0.05)$ overlapped (Additional file 1 : Table S13; Additional file 4: Figure S4). A proxy of rs6750142 (rs726430, $r^{2}=1$ ) associated with methylation at cg07547695 was nominally associated ( $p$-value $<0.05)$ with both CHD and T2D in GWAS $[18,20]$.

We investigated the behaviour of 1599 significant meQTL SNPs $(F D R<0.05)$ in GWAS data from the CARDIoGRAM consortium for CHD [20] and the DIAGRAM consortium for T2D [18]. We found no evidence of overrepresentation of nominally significant associations ( $p$-value $<0.05$ ) for CHD (one-sided Fisher exact $p$ value $=0.99$ ) or T2D (one-sided Fisher exact $p$-value $=$ 0.93) among significant meQTL SNPs. One or more significant meQTL SNPs of nine CpG sites were nominally associated with CHD in GWAS data from the CARDIo GRAM consortium ( $p$-value $<0.05)$. These were annotated to AMDHD1 (cg15351688: rs10777751, rs7955450, rs7486703), BCL2L11 (cg07547695: rs726430), FAM20C (cg05969038: rs7786461; cg21913519: rs7786461), HSP9 OAB1 (cg26241416: rs6905285, rs7758726, rs666462), SEMA6A (cg06650664: rs10077506, rs17139825), WNK2 (cg14396800: rs2991377,rs10992689, rs10821105) and intergenic sites on chr. 5 (cg15609272: rs17057846) and chr. 12 (cg17173663: rs1354156) (Additional file 5). In analogous analysis on GWAS data from the DIAGRAM consortium, we found nominal associations for SNPs associated with methylation at CpG sites annotated to BCL2L11 (cg07547695: rs726430), CEP55 (cg24371383: rs12782691), CPB1 (cg04072156: rs16861015) and an intergenic site on chr. 4 (cg14532755: rs1665364, rs2236 786, rs3752749, rs732754, rs744658, rs798719, rs798726, rs798727, rs798741, rs798744, rs798751, rs798754, rs79 8755 , rs798756, rs798766, rs811316, rs8389) ( $p$-value < 0.05; Additional file 5).

The relationship between oxidative stress-associated CpGs and disease incidence

As markers of oxidative stress have been associated with both CVD and T2D, we sought to assess the role of epigenetics in this process. Methylation levels at the 66 CpGs associated with oxidative markers were tested for association with incident CVD $(n=180)$ and T2D $(n=$ 71) events during a 10-year follow-up using logistic regression models to examine their potential role in disease. Epigenetic variation in the 3'-UTR of HIST1H4D (cg08170869; histone cluster 1, H4d) and in the body of DVL1 (cg03465880; dishevelled-1) were associated with incident T2D (permutation $p$-value $<0.01$; Table 2). For every SD decrease in methylation $\beta$ value at $\operatorname{cg} 08170869$, the risk of T2D was $39 \%$ higher (nominal $p$-value $=$ 0.0034; permutation $p$-value $=0.0044$; odds ratio $[\mathrm{OR}]$ per SD decrement $=1.39$ [95\% CI, 1.15-1.57]). Similarly, hypomethylation in $D V L 1$ was associated with higher risk of T2D (nominal $p$-value $=0.0073$; permutation $p$-value $=$ 0.0080; OR per SD decrement $=1.31$ [95\% CI, 1.10-1.47]).

Analogous analysis of CVD revealed no significant association of methylation levels at the phenotypeassociated CpGs with incident CVD events (permutation $p$-value $<0.01)$. The strongest association was to methylation levels at cg11842944 in the body of ZNF341 (nominal $p$-value $=0.013$; permutation $p$-value $=0.016$; OR per SD decrement $=1.23$ [95\% CI, 1.05-1.42]). 
Table 2 Association of oxidative marker-associated CpGs with incident T2D ${ }^{\mathrm{a}}$ events (permutation $p$-value $<0.01$ )

\begin{tabular}{|c|c|c|c|c|c|c|c|c|c|}
\hline \multirow[b]{2}{*}{$\begin{array}{l}\text { Disease } \\
\text { outcome }\end{array}$} & \multirow[b]{2}{*}{ CpG } & \multirow[b]{2}{*}{ Gene } & \multirow[b]{2}{*}{ Description } & \multirow[b]{2}{*}{$\begin{array}{l}\text { Gene } \\
\text { Property }\end{array}$} & \multicolumn{3}{|l|}{ Primary model } & \multirow{2}{*}{$\begin{array}{l}\text { Secondary } \\
\text { model }^{d} \\
P \text {-value }\end{array}$} & \multirow[b]{2}{*}{$\begin{array}{l}\text { Oxidative marker } \\
\text { association (Direction) }\end{array}$} \\
\hline & & & & & $\begin{array}{l}\text { OR } \\
(95 \% \mathrm{Cls})^{\mathrm{b}}\end{array}$ & $\begin{array}{l}\text { Nominal } \\
P \text {-value }\end{array}$ & $\begin{array}{l}\text { Permutation } \\
P \text {-value }\end{array}$ & & \\
\hline $\mathrm{T} 2 \mathrm{D}$ & cg08170869 & HISTIHAD & Histone cluster $1, \mathrm{H} 4 \mathrm{~d}$ & 3'-UTR & $1.39(1.15-1.57)$ & $3.39 \mathrm{E}-03$ & $4.40 \mathrm{E}-03$ & 9.19E-03 & GSH (+) \\
\hline $\mathrm{T} 2 \mathrm{D}$ & cg03465880 & DVL1 & $\begin{array}{l}\text { Dishevelled segment } \\
\text { polarity protein } 1\end{array}$ & Body & $1.31(1.10-1.47)$ & $7.27 \mathrm{E}-03$ & $8.00 \mathrm{E}-03$ & $6.48 \mathrm{E}-03$ & GSH (-) \\
\hline
\end{tabular}

T2D type 2 diabetes, $C p G$ cytosine-guanine site, OR odds ratio, $\mathrm{Cl}$ confidence intervals, GSH reduced glutathione, $S D$ standard deviation

${ }^{a}$ Events up to 10 years after baseline; number of $\mathrm{T} 2 \mathrm{D}$ events $=71$

${ }^{b}$ Corresponds to the OR increase in risk of disease per SD decrement in DNA methylation

${ }^{c} P$-value from permutation test ( $n=10,000$ permutations)

${ }^{\mathrm{d}}$ Secondary model also included BMI and smoking

e Direction of association between oxidative marker(s) and methylation at CpG site. A + symbol corresponds to that increased levels of DNA methylation associate with increased levels of the oxidative marker

Analysing myocardial infarction $(n=55)$, heart failure $(n$ $=76)$ and ischaemic stroke $(n=47)$ separately, methylation at this $\mathrm{CpG}$ showed the strongest association to heart failure (nominal $p$-value $=0.0087$; permutation $p$ value $=0.0126 ; \mathrm{OR}$ per $\mathrm{SD}$ decrement $=1.28[95 \% \mathrm{CI}$, $1.06-1.50])$. This gene lies in a region previously associated with height in GWAS [62] and encodes a gene product involved in transcriptional regulation.

\section{Discussion}

Oxidative stress has previously been associated with development of cardiometabolic disease. In this study, the role of epigenetic changes in blood cells for oxidative stress and development of CVD and T2D was examined through analyses of genome-wide DNA methylation data and ten markers of oxidative stress, CVD and T2D in up to 966 individuals of the same age.

We identified numerous blood CpGs for which levels of methylation correlated with markers of oxidative stress. Enrichment of associations to DNA methylation in $\mathrm{CpG}$ island shores, previously noted to be dynamic $[63,64]$ and correlated with gene expression [63], indicates that differential methylation with oxidative stress may play a role in transcriptional regulation. Overlap with functional regulatory elements for one-fourth of associated CpGs support a functional role for these methylation changes with oxidative stress. Enrichment of TF binding sites in regions upstream of genes annotated to phenotype-associated CpGs indicates a common set of regulatory signals. TFs included E2F1 with prior evidence of a function in processes relating to atherosclerosis and CHD [60], connecting genes for which CpG methylation changed with oxidative stress to CVD.

Methylation variation in the 3'-UTR of HIST1H4D (cg08170869) and in the body of DVL1 (cg03465880) associated with GSH were also associated with incident T2D. Previous evidence indicates changes in levels of GSH in individuals with T2D $[65,66]$. Evidence from previous studies implicates $D V L 1$ in processes related to T2D [67]. DVL1 encodes a gene product with an important role in Wnt signalling, important in adipogeneis, and has, for example, been found to be downregulated in adipocytes from non-obese insulin resistant individuals compared to controls [67].

Roughly forty percent of oxidative stress-associated CpGs were regulated by genetic sequence variation in cis. We found evidence of genotype-phenotype associations acting via epigenetic variation at gene BCL2L11 (BCL2-like 11 [apoptosis facilitator]). Genetic variants close to this gene have previously been associated with the biological ageing marker dehydroepiandrosterone sulphate in GWAS [68]. Integration of meQTLs with GWAS data on CHD and T2D showed no significant enrichment in nominal associations for meQTL SNPs of oxidative stress-associated CpGs.

Previous evidence indicates a role for some of the genes annotated to oxidative stress marker-associated CpGs in metabolic or cardiovascular disease, indicating that epigenetic changes with oxidative stress may reflect important disease processes. Lipid-related oxidative markers such as oxLDL has previously been shown to play a role in atherosclerosis $[8,9]$ and to associate with insulin resistance [10]. In agreement with this, we found methylation at genes involved in some of these processes to associate with lipid-related oxidative markers. $R G L 2$ (for which promoter methylation was higher with higher $\mathrm{CD})$ has been shown to have a protective role in response to cardiac stress in vitro [69]. Furthermore, previous results implicate RGL2 in atherosclerosis pathogenesis. RGL2 in complex with SAMD9 have an inhibitory function on expression of the transcription factor $E G R 1$, which is highly expressed in atherosclerotic lesions and has been shown to be involved in induction of the coagulation protein tissue factor in response to oxLDL [70-72]. Earlier studies suggest a role of the transcription factor FOSL2 (for which intragenic methylation was higher with BCD-LDL) in processes relating to cardiac fibrosis of ischaemic tissue through its oxygensensitive induction of TGF $\beta$ in cardiac fibroblasts [73]. FOSL2 also regulates leptin expression in adipocytes [74]. 
The association of BCD-LDL to promoter methylation of this gene in blood was not driven by obesity as the signal still remained following adjustment for BMI. Additionally, genetic studies of QRS duration [75], which has been associated with increased risk of heart failure [76, 77], indicate a role of LRIG1. In our study, methylation at this gene associated with $\mathrm{HCY}$, an amino acid previously associated with inflammation in blood vessels, progression to atherosclerosis and development of CVD, particularly ischemic stroke $[11,12]$. However, the causal role of homocysteine in CVD has been challenged [13-15].

The main strengths of the present study include the large sample size that underwent measurements of genome-wide DNA methylation, ten markers of reflecting different aspects of oxidative stress and the 10 years of follow-up allowing analyses of incident disease endpoints. The study also has some limitations. First, we acknowledge that the most important limitation is the lack of replication of oxidative stress-associated DNA methylation. To our knowledge, there are no other study samples with the needed data available making replication impossible, but we have done our best to avoid false positive findings via strict correction for multiple testing and integration with data on gene function, regulation and on related phenotypes from external data sources. Second, gene expression data for the same individuals were not available to assess the effect of epigenetic variation with oxidative markers on transcription in blood. Third, while blood is easily accessible and thus attractive for biomarker discovery, clinical diagnostics and translation, blood derived cells may not be the most relevant tissue for drawing biological conclusions about oxidative stress, CVD and T2D. However, previous studies have shown methylation variation in blood to be a good proxy of differential methylation in various tissues [33-37].

\section{Conclusions}

We found novel epigenetic changes in blood to be associated with markers of oxidative stress, two of these with evidence of a relation to T2D. Further studies are needed to replicate the findings on DNA methylation with oxidative stress, as well as determining the effect of epigenetic variation related to oxidative stress on downstream molecular biological phenotypes.

\section{Additional files}

Additional file 1: Supplementary Data. Figure S1. Plot showing the first two PC components of the PIVUS genotype data with the $1000 \mathrm{G}$ multi population reference panel. Figure S3. Comparison of regression coefficients from the primary and secondary models (additionally adjusted for BMI) for oxidative marker BCD-LDL. Table S9. Enrichment in JASPAR transcription factor binding site motifs in genes annotated to oxidative stress associated CpGs (Bonferroni-adjusted $p$-value $<0.05$ ).
Table S10. Enriched biological process among genes annotated to oxidative marker associated CpGs (adjusted $p$-value $<0.05$ ). Table S11. Enriched annotation clusters among genes annotated to oxidative marker CpGs (enrichment score > 1). Table S12. Significant lead cis-meQTL SNPS of oxidative marker CpGs (FDR <0.05). Table S13. Overlap across genotype-CpG (FDR $<0.05)$, genotype-phenotype ( $p$-value $<0.001$ ), and CpG-phenotype (FDR <0.05) results. (DOCX $135 \mathrm{~kb}$ )

Additional file 2: Figure S2. DNA methylation sites associated with oxidative markers at a Bonferroni-corrected alpha threshold 0.05 ( $p$-value $<1.1 \mathrm{E}-07$ ). $\mathrm{CpG}$ sites are ordered by chromosomal position from bottom (chr. 1) to top (chr. 22). (EPS $14 \mathrm{~kb}$ )

Additional file 3: Supplemental Tables. Table S1. Methylation sites associated with TGSH (FDR <0.05). Table S2. Methylation sites associated with GSH (FDR $<0.05$. Table S3. Methylation sites associated with GSSG (FDR $<0.05$ ). Table S4. Methylation sites associated with ratio of GSSG-to-GSH (FDR $<0.05)$. Table S5. Methylation sites associated with levels of HCY (FDR <0.05). Table S6. Methylation sites associated with levels of oxLDL (FDR <0.05). Table S7. Methylation sites associated with levels of $C D(F D R<0.05)$. Table S8: Methylation sites associated with BCD-LDL (FDR <0.05). (XLS $185 \mathrm{~kb}$ )

Additional file 4: Figure S4. Overview of the analysis of genetic associations of oxidative stress-associated DNA methylation. The diagram shaded in blue depicts the associations at the two instances where we observed an overlap across genotype-CpG (FDR $<0.05)$, genotypephenotype $(p<0.001)$, and CpG-phenotype (FDR < 0.05). (XLSX $60 \mathrm{~kb})$

Additional file 5: Table S14. Nominal significant associations ( $p$-value $<0.05$ ) for significant meQTL SNPs in GWAS data from the CARDIOGRAMplusC4D and DIAGRAM consortia. (XLSX $59 \mathrm{~kb}$ )

\section{Abbreviations}

BCD-LDL: Baseline conjugated dienes LDL; BMI: Body mass index;; CD: Conjugated dienes; CHD: Coronary heart disease; CpG: Cytosine-guanine; CVD: Cardiovascular disease; FDR: False discovery rate; GSH: Reduced glutathione; GSSG: Oxidised glutathione; GWAS: Genome-wide association studies; HCY: Homocysteine; LDL: Low-density lipoprotein; MAF: Minor allele frequency; meQTL: Methylation quantitative trait loci; OLAB: Antibodies against oxLDL; OxLDL: Oxidised LDL; PIVUS: The Prospective Investigation of the Vasculature in Uppsala Seniors; SD: Standard deviation; T2D: Type 2 diabetes; TAOC: Total antioxidant capacity; TGSH: Total glutathione; TSS: Transcriptional start site

\section{Acknowledgements}

Data on coronary artery disease / myocardial infarction have been contributed by CARDIoGRAMplusC4D investigators and were downloaded from http://www.cardiogramplusc4d.org/. Data on T2D have been contributed by DIAGRAM investigators and were downloaded from http://diagram-consortium.org/downloads.html. We thank Dr. Stefan Gustafsson for help with pre-processing and quality control of DNA methylation data.

\section{Funding}

El receives funding from Knut och Alice Wallenberg Foundation, Swedish Research Council (VR; grant no. 2012-1397) and Swedish Heart-Lung Foundation (20120197). ÅKH receives funding from Linne Stiftelsen for the project. Genome-wide DNA methylation profiling was funded by the Uppsala University Hospital (ALF-medel) and was performed by the SNP\&SEQ Technology Platform in Uppsala. The facility is part of the National Genomics Infrastructure (NGI) Sweden and Science for Life Laboratory. The SNP\&SEQ Platform is also supported by the Swedish Research Council and the Knut and Alice Wallenberg Foundation. M.Z. was supported by a grant from the Estonian Research Council IUT 20-42 and by the EU through the European Regional Development Fund (Project No. 2014-2020.4.01.15-0012).

\section{Authors' contributions}

$\AA \AA \mathrm{KH}, \mathrm{LL}$ and El conceived and designed the study. ÅKH performed statistical analyses and wrote the manuscript. El supervised the project; reviewed and revised the manuscript. MZ performed or supervised collection of oxidative marker phenotype data. LL and JS performed or supervised clinical data collection, phenotyping and/or methylation profiling. All authors read, provided feedback and approved the final manuscript. 


\section{Competing interests}

Erik Ingelsson is an advisor and consultant for Precision Wellness Inc., and an advisor for Cellink.

The other authors declare that they have no competing interests.

\section{Consent for publication}

Not applicable.

\section{Ethics approval and consent to participate}

Ethical approval for the project was granted by the Ethics Committee at Uppsala University, Sweden (reference number 00-419) and all samples were collected after obtaining written and signed informed consent.

\section{Author details}

${ }^{1}$ Department of Medical Sciences, Molecular Epidemiology and Science for Life Laboratory, Uppsala University, Uppsala, Sweden. ${ }^{2}$ Institute of Biomedicine and Translational Medicine, Department of Biochemistry, The Centre of Excellence for Genomics and Translational Medicine, University of Tartu, Tartu, Estonia. ${ }^{3}$ Department of Medical Sciences, Cardiovascular Epidemiology, Uppsala University Hospital, Uppsala, Sweden. ${ }^{4}$ Department of Medicine, Division of Cardiovascular Medicine, Stanford University School of Medicine, Stanford, CA, USA.

Received: 6 August 2016 Accepted: 14 November 2016 Published online: 25 November 2016

\section{References}

1. Mendis S, Puska P, Norrving B. Global atlas on cardiovascular disease prevention and control. Geneva: World Health Organization; 2011.

2. Keaney Jr JF, Larson MG, Vasan RS, Wilson PW, Lipinska I, Corey D, Massaro JM, Sutherland P, Vita JA, Benjamin EJ. Obesity and systemic oxidative stress: clinical correlates of oxidative stress in the Framingham Study. Arterioscler Thromb Vasc Biol. 2003;23(3):434-9.

3. Stephens JW, Khanolkar MP, Bain SC. The biological relevance and measurement of plasma markers of oxidative stress in diabetes and cardiovascular disease. Atherosclerosis. 2009;202(2):321-9.

4. Harrison D, Griendling KK, Landmesser U, Hornig B, Drexler H. Role of oxidative stress in atherosclerosis. Am J Cardiol. 2003;91(3A):7A-11.

5. Lind L, Andersson J, Ronn M, Gustavsson T, Holdfelt P, Hulthe J, Elmgren A, Zilmer K, Zilmer M. Brachial artery intima-media thickness and echogenicity in relation to lipids and markers of oxidative stress in elderly subjects:-the prospective investigation of the vasculature in Uppsala Seniors (PIVUS) Study. Lipids. 2008;43(2):133-41.

6. Holvoet P, Mertens A, Verhamme P, Bogaerts K, Beyens G, Verhaeghe R, Collen D, Muls E, Van de Werf F. Circulating oxidized LDL is a useful marker for identifying patients with coronary artery disease. Arterioscler Thromb Vasc Biol. 2001;21(5):844-8.

7. Holvoet $P$, Jenny NS, Schreiner PJ, Tracy RP, Jacobs DR. The relationship between oxidized LDL and other cardiovascular risk factors and subclinical CVD in different ethnic groups: the Multi-Ethnic Study of Atherosclerosis (MESA). Atherosclerosis. 2007;194(1):245-52.

8. Frostegard J, Nilsson J, Haegerstrand A, Hamsten A, Wigzell H, Gidlund M. Oxidized low density lipoprotein induces differentiation and adhesion of human monocytes and the monocytic cell line U937. Proc Natl Acad Sci U S A. 1990;87(3):904-8.

9. Matsuura E, Kobayashi K, Tabuchi M, Lopez LR. Oxidative modification of low-density lipoprotein and immune regulation of atherosclerosis. Prog Lipid Res. 2006;45(6):466-86.

10. Park K, Gross M, Lee DH, Holvoet P, Himes JH, Shikany JM, Jacobs Jr DR. Oxidative stress and insulin resistance: the coronary artery risk development in young adults study. Diabetes Care. 2009;32(7):1302-7.

11. Cotlarciuc I, Malik R, Holliday EG, Ahmadi KR, Pare G, Psaty BM, Fornage M, Hasan N, Rinne PE, Ikram MA, et al. Effect of genetic variants associated with plasma homocysteine levels on stroke risk. Stroke. 2014;45(7):1920-4.

12. Park JH, Saposnik G, Ovbiagele B, Markovic D, Towfighi A. Effect of Bvitamins on stroke risk among individuals with vascular disease who are not on antiplatelets: A meta-analysis. Int J Stroke. 2016;11(2):206-11.

13. Clarke R, Halsey J, Lewington S, Lonn E, Armitage J, Manson JE, Bonaa KH, Spence JD, Nygard O, Jamison R, et al. Effects of lowering homocysteine levels with B vitamins on cardiovascular disease, cancer, and cause-specific mortality: Meta-analysis of 8 randomized trials involving 37485 individuals. Arch Intern Med. 2010;170(18):1622-31.

14. Clarke R, Bennett DA, Parish S, Verhoef P, Dotsch-Klerk M, Lathrop M, Xu P, Nordestgaard BG, Holm H, Hopewell JC, et al. Homocysteine and coronary heart disease: meta-analysis of MTHFR case-control studies, avoiding publication bias. PLoS Med. 2012;9(2), e1001177.

15. Marti-Carvajal AJ, Sola I, Lathyris D. Homocysteine-lowering interventions for preventing cardiovascular events. Cochrane Database Syst Rev. 2015;1, CD006612.

16. Dupuis J, Langenberg C, Prokopenko I, Saxena R, Soranzo N, Jackson AU, Wheeler E, Glazer NL, Bouatia-Naji N, Gloyn AL, et al. New genetic loci implicated in fasting glucose homeostasis and their impact on type 2 diabetes risk. Nat Genet. 2010;42(2):105-16.

17. Saxena R, Hivert MF, Langenberg C, Tanaka T, Pankow JS, Vollenweider P, Lyssenko V, Bouatia-Naji N, Dupuis J, Jackson AU, et al. Genetic variation in GIPR influences the glucose and insulin responses to an oral glucose challenge. Nat Genet. 2010;42(2):142-8.

18. Morris AP, Voight BF, Teslovich TM, Ferreira T, Segre AV, Steinthorsdottir V, Strawbridge RJ, Khan H, Grallert H, Mahajan A, et al. Large-scale association analysis provides insights into the genetic architecture and pathophysiology of type 2 diabetes. Nat Genet. 2012;44(9):981-90.

19. Scott RA, Lagou V, Welch RP, Wheeler E, Montasser ME, Luan J, Magi R, Strawbridge RJ, Rehnberg E, Gustafsson S, et al. Large-scale association analyses identify new loci influencing glycemic traits and provide insight into the underlying biological pathways. Nat Genet. 2012;44(9): 991-1005.

20. Deloukas P, Kanoni S, Willenborg C, Farrall M, Assimes TL, Thompson JR, Ingelsson E, Saleheen D, Erdmann J, Goldstein BA, et al. Large-scale association analysis identifies new risk loci for coronary artery disease. Nat Genet. 2013;45(1):25-33.

21. Wolffe AP, Guschin D. Chromatin structural features and targets that regulate transcription. J Struct Biol. 2000;129(2-3):102-22.

22. Rakyan VK, Down TA, Balding DJ, Beck S. Epigenome-wide association studies for common human diseases. Nat Rev Genet. 2011;12(8):529-41.

23. Feinberg AP, Irizarry RA, Fradin D, Aryee MJ, Murakami P, Aspelund T, Eiriksdottir G, Harris TB, Launer L, Gudnason V, et al. Personalized epigenomic signatures that are stable over time and covary with body mass index. Sci Transl Med. 2010;2(49):49-67.

24. Dick KJ, Nelson CP, Tsaprouni L, Sandling JK, Aissi D, Wahl S, Meduri E, Morange PE, Gagnon F, Grallert H, et al. DNA methylation and body-mass index: a genome-wide analysis. Lancet. 2014;383(9933):1990-8.

25. Jones PA, Baylin SB. The fundamental role of epigenetic events in cancer. Nat Rev Genet. 2002;3(6):415-28.

26. Gervin K, Vigeland MD, Mattingsdal M, Hammero M, Nygard H, Olsen AO, Brandt I, Harris JR, Undlien DE, Lyle R. DNA methylation and gene expression changes in monozygotic twins discordant for psoriasis: identification of epigenetically dysregulated genes. PLoS Genet. 2012;8(1), e1002454.

27. Shenker NS, Polidoro S, van Veldhoven K, Sacerdote C, Ricceri F, Birrell MA, Belvisi MG, Brown R, Vineis P, Flanagan JM. Epigenome-wide association study in the European Prospective Investigation into Cancer and Nutrition (EPIC-Turin) identifies novel genetic loci associated with smoking. Hum Mol Genet. 2013;22(5):843-51.

28. Zeilinger S, Kuhnel B, Klopp N, Baurecht H, Kleinschmidt A, Gieger C, Weidinger S, Lattka E, Adamski J, Peters A, et al. Tobacco smoking leads to extensive genome-wide changes in DNA methylation. PLoS One. 2013;8(5), e63812.

29. Campos AC, Molognoni F, Melo FH, Galdieri LC, Carneiro CR, D'Almeida V, Correa $M$, Jasiulionis MG. Oxidative stress modulates DNA methylation during melanocyte anchorage blockade associated with malignant transformation. Neoplasia. 2007;9(12):1111-21.

30. Zawia NH, Lahiri DK, Cardozo-Pelaez F. Epigenetics, oxidative stress, and Alzheimer disease. Free Radic Biol Med. 2009;46(9):1241-9.

31. Wrigley BJ, Lip GY, Shantsila E. The role of monocytes and inflammation in the pathophysiology of heart failure. Eur J Heart Fail. 2011;13(11):1161-71.

32. Jagannathan-Bogdan M, McDonnell ME, Shin H, Rehman Q, Hasturk H, Apovian CM, Nikolajczyk BS. Elevated proinflammatory cytokine production by a skewed $T$ cell compartment requires monocytes and promotes inflammation in type 2 diabetes. J Immunol. 2011;186(2):1162-72.

33. Bacos K, Gillberg L, Volkov P, Olsson AH, Hansen T, Pedersen O, Gjesing AP, Eiberg H, Tuomi T, Almgren P, et al. Blood-based biomarkers of ageassociated epigenetic changes in human islets associate with insulin secretion and diabetes. Nat Commun. 2016;7:11089. 
34. Lehmann-Werman R, Neiman D, Zemmour H, Moss J, Magenheim J, VakninDembinsky A, Rubertsson S, Nellgard B, Blennow K, Zetterberg H, et al. Identification of tissue-specific cell death using methylation patterns of circulating DNA. Proc Natl Acad Sci U S A. 2016;113(13):E1826-34.

35. Walton E, Hass J, Liu J, Roffman JL, Bernardoni F, Roessner V, Kirsch M, Schackert G, Calhoun V, Ehrlich S. Correspondence of DNA methylation between blood and brain tissue and its application to schizophrenia research. Schizophr Bull. 2016;42(2):406-14.

36. Hardy T, Zeybel M, Day CP, Dipper C, Masson S, McPherson S, Henderson E, Tiniakos D, White S, French J, et al. Plasma DNA methylation: a potential biomarker for stratification of liver fibrosis in non-alcoholic fatty liver disease. Gut. 2016. doi: 10.1136/gutjnl-2016-311526.

37. Huang YT, Chu S, Loucks EB, Lin CL, Eaton CB, Buka SL, Kelsey KT: Epigenome-wide profiling of DNA methylation in paired samples of adipose tissue and blood. Epigenetics. 2016; 11(3):227-36

38. Lind L, Fors N, Hall J, Marttala K, Stenborg A. A comparison of three different methods to evaluate endothelium-dependent vasodilation in the elderly: the Prospective Investigation of the Vasculature in Uppsala Seniors (PIVUS) study. Arterioscler Thromb Vasc Biol. 2005:25(11):2368-75.

39. Annuk M, Zilmer M, Lind L, Linde T, Fellstrom B. Oxidative stress and endothelial function in chronic renal failure. J Am Soc Nephrol. 2001;12(12):2747-52.

40. Ahotupa M, Marniemi J, Lehtimaki T, Talvinen K, Raitakari OT, Vasankari T, Viikari J, Luoma J, Yla-Herttuala S. Baseline diene conjugation in LDL lipids as a direct measure of in vivo LDL oxidation. Clin Biochem. 1998;31(4):257-61.

41. Grundberg E, Meduri E, Sandling JK, Hedman AK, Keildson S, Buil A, Busche S, Yuan W, Nisbet J, Sekowska M, et al. Global analysis of DNA methylation variation in adipose tissue from twins reveals links to disease-associated variants in distal regulatory elements. Am J Hum Genet. 2013;93(5):876-90.

42. Bibikova M, Barnes B, Tsan C, Ho V, Klotzle B, Le JM, Delano D, Zhang L, Schroth GP, Gunderson KL, et al. High density DNA methylation array with single CpG site resolution. Genomics. 2011;98(4):288-95.

43. Marchini J, Howie B, Myers S, McVean G, Donnelly P. A new multipoin method for genome-wide association studies by imputation of genotypes. Nat Genet. 2007;39(7):906-13.

44. Houseman EA, Accomando WP, Koestler DC, Christensen BC, Marsit CJ, Nelson $\mathrm{HH}$, Wiencke JK, Kelsey KT. DNA methylation arrays as surrogate measures of cell mixture distribution. BMC Bioinformatics. 2012;13:86.

45. Aryee MJ, Jaffe AE, Corrada-Bravo H, Ladd-Acosta C, Feinberg AP, Hansen KD, Irizarry RA. Minfi: a flexible and comprehensive Bioconductor package for the analysis of Infinium DNA methylation microarrays. Bioinformatics. 2014;30(10):1363-9.

46. Reinius LE, Acevedo N, Joerink M, Pershagen G, Dahlen SE, Greco D, Soderhall C, Scheynius A, Kere J. Differential DNA methylation in purified human blood cells: implications for cell lineage and studies on disease susceptibility. PLoS One. 2012;7(7), e41361.

47. Team RC. R: A language and environment for statistical computing. Vienna: R Foundation for Statistical Computing; 2012.

48. Storey JD, Tibshirani R. Statistical significance for genomewide studies. Proc Natl Acad Sci U S A. 2003;100(16):9440-5.

49. Li Y, Willer CJ, Ding J, Scheet P, Abecasis GR. MaCH: using sequence and genotype data to estimate haplotypes and unobserved genotypes. Genet Epidemiol. 2010;34(8):816-34.

50. Boyle AP, Hong EL, Hariharan M, Cheng Y, Schaub MA, Kasowski M, Karczewski KJ, Park J, Hitz BC, Weng S, et al. Annotation of functional variation in personal genomes using RegulomeDB. Genome Res. 2012;22(9):1790-7.

51. Zambelli F, Pesole G, Pavesi G. Pscan: finding over-represented transcription factor binding site motifs in sequences from co-regulated or co-expressed genes. Nucleic Acids Res. 2009;37(Web Server issue):W247-52.

52. Portales-Casamar E, Thongjuea S, Kwon AT, Arenillas D, Zhao X, Valen E, Yusuf D, Lenhard B, Wasserman WW, Sandelin A. JASPAR 2010: the greatly expanded open-access database of transcription factor binding profiles. Nucleic Acids Res. 2010;38(Database issue):D105-10.

53. da Huang W, Sherman BT, Lempicki RA. Systematic and integrative analysis of large gene lists using DAVID bioinformatics resources. Nat Protoc. 2009:4(1):44-57.

54. da Huang W, Sherman BT, Lempicki RA. Bioinformatics enrichment tools: paths toward the comprehensive functional analysis of large gene lists. Nucleic Acids Res. 2009;37(1):1-13.

55. Thomas PD, Kejariwal A, Guo N, Mi H, Campbell MJ, Muruganujan A, Lazareva-Ulitsky B. Applications for protein sequence-function evolution data: mRNA/protein expression analysis and coding SNP scoring tools. Nucleic Acids Res. 2006;34(Web Server issue):W645-50.

56. Mi H, Muruganujan A, Thomas PD. PANTHER in 2013: modeling the evolution of gene function, and other gene attributes, in the context of phylogenetic trees. Nucleic Acids Res. 2013;41(Database issue):D377-86.

57. Nikolsky Y, Bryant J. Protein networks and pathway analysis. Preface. Methods Mol Biol. 2009;563:v-vii.

58. Dhanabal M, Wu F, Alvarez E, McQueeney KD, Jeffers M, MacDougall J, Boldog FL, Hackett C, Shenoy S, Khramtsov N, et al. Recombinant semaphorin 6A-1 ectodomain inhibits in vivo growth factor and tumor cell line-induced angiogenesis. Cancer Biol Ther. 2005;4(6):659-68.

59. Urbich C, Kaluza D, Fromel T, Knau A, Bennewitz K, Boon RA, Bonauer A, Doebele C, Boeckel JN, Hergenreider E, et al. MicroRNA-27a/b controls endothelial cell repulsion and angiogenesis by targeting semaphorin 6A. Blood. 2012;119(6):1607-16.

60. Pilbrow AP, Folkersen L, Pearson JF, Brown CM, McNoe L, Wang NM, Sweet WE, Tang WH, Black MA, Troughton RW, et al. The chromosome 9p21.3 coronary heart disease risk allele is associated with altered gene expression in normal heart and vascular tissues. PLoS One. 2012;7(6):e39574.

61. Bell JT, Pai AA, Pickrell JK, Gaffney DJ, Pique-Regi R, Degner JF, Gilad Y, Pritchard JK. DNA methylation patterns associate with genetic and gene expression variation in HapMap cell lines. Genome Biol. 2011;12(1):R10.

62. Lango Allen H, Estrada K, Lettre G, Berndt SI, Weedon MN, Rivadeneira F, Willer CJ, Jackson AU, Vedantam S, Raychaudhuri S, et al. Hundreds of variants clustered in genomic loci and biological pathways affect human height. Nature. 2010;467(7317):832-8.

63. Irizarry RA, Ladd-Acosta C, Wen B, Wu Z, Montano C, Onyango P, Cui H, Gabo K, Rongione M, Webster M, et al. The human colon cancer methylome shows similar hypo- and hypermethylation at conserved tissuespecific CpG island shores. Nat Genet. 2009;41(2):178-86.

64. Ziller MJ, Gu H, Muller F, Donaghey J, Tsai LT, Kohlbacher O, De Jager PL, Rosen ED, Bennett DA, Bernstein BE, et al. Charting a dynamic DNA methylation landscape of the human genome. Nature. 2013;500(7463):477-81.

65. Calabrese V, Cornelius C, Leso V, Trovato-Salinaro A, Ventimiglia B, Cavallaro M, Scuto M, Rizza S, Zanoli L, Neri S, et al. Oxidative stress, glutathione status, sirtuin and cellular stress response in type 2 diabetes. Biochim Biophys Acta. 2012;1822(5):729-36.

66. Jain SK, Micinski D, Huning L, Kahlon G, Bass PF, Levine SN. Vitamin D and Lcysteine levels correlate positively with GSH and negatively with insulin resistance levels in the blood of type 2 diabetic patients. Eur J Clin Nutr. 2014;68(10):1148-53.

67. Yang X, Jansson PA, Nagaev I, Jack MM, Carvalho E, Sunnerhagen KS, Cam MC, Cushman SW, Smith U. Evidence of impaired adipogenesis in insulin resistance. Biochem Biophys Res Commun. 2004;317(4):1045-51.

68. Zhai G, Teumer A, Stolk L, Perry JR, Vandenput L, Coviello AD, Koster A, Bell JT, Bhasin S, Eriksson J, et al. Eight common genetic variants associated with serum DHEAS levels suggest a key role in ageing mechanisms. PLoS Genet. 2011;7(4), e1002025.

69. Scotland RL, Allen L, Hennings LJ, Post GR, Post SR. The ral exchange factor rgl2 promotes cardiomyocyte survival and inhibits cardiac fibrosis. PLoS One. 2013;8(9), e73599.

70. Cui MZ, Penn MS, Chisolm GM. Native and oxidized low density lipoprotein induction of tissue factor gene expression in smooth muscle cells is mediated by both Egr-1 and Sp1. J Biol Chem. 1999;274(46):32795-802.

71. McCaffrey TA, Fu C, Du B, Eksinar S, Kent KC, Bush Jr H, Kreiger K, Rosengart T, Cybulsky MI, Silverman ES, et al. High-level expression of Egr-1 and Egr-1-inducible genes in mouse and human atherosclerosis. J Clin Invest. 2000;105(5):653-62.

72. Hershkovitz D, Gross Y, Nahum S, Yehezkel S, Sarig O, Uitto J, Sprecher E. Functional characterization of SAMD9, a protein deficient in normophosphatemic familial tumoral calcinosis. J Invest Dermatol. 2011;131(3):662-9.

73. Roy S, Khanna S, Azad A, Schnitt R, He G, Weigert C, Ichijo H, Sen CK. Fra-2 mediates oxygen-sensitive induction of transforming growth factor beta in cardiac fibroblasts. Cardiovasc Res. 2010;87(4):647-55.

74. Wrann CD, Eguchi J, Bozec A, Xu Z, Mikkelsen T, Gimble J, Nave H, Wagner $E F$, Ong SE, Rosen ED. FOSL2 promotes leptin gene expression in human and mouse adipocytes. J Clin Invest. 2012;122(3):1010-21.

75. Sotoodehnia N, Isaacs A, de Bakker PI, Dorr M, Newton-Cheh C, Nolte IM, van der Harst $P$, Muller M, Eijgelsheim M, Alonso A, et al. Common variants in 22 loci are associated with QRS duration and cardiac ventricular conduction. Nat Genet. 2010;42(12):1068-76. 
76. Dhingra R, Pencina MJ, Wang TJ, Nam BH, Benjamin EJ, Levy D, Larson MG, Kannel WB, D'Agostino Sr RB, Vasan RS. Electrocardiographic QRS duration and the risk of congestive heart failure: the Framingham Heart Study. Hypertension. 2006:47(5):861-7.

77. Ilkhanoff L, Liu K, Ning H, Nazarian S, Bluemke DA, Soliman EZ, Lloyd-Jones DM. Association of QRS duration with left ventricular structure and function and risk of heart failure in middle-aged and older adults: the Multi-Ethnic Study of Atherosclerosis (MESA). Eur J Heart Fail. 2012;14(11):1285-92.

Submit your next manuscript to BioMed Central and we will help you at every step:

- We accept pre-submission inquiries

- Our selector tool helps you to find the most relevant journal

- We provide round the clock customer support

- Convenient online submission

- Thorough peer review

- Inclusion in PubMed and all major indexing services

- Maximum visibility for your research

Submit your manuscript at www.biomedcentral.com/submit 